\title{
Insect galls of the xeric vegetation of Ilha do Cabo Frio (Arraial do Cabo, RJ, Brazil)
}

\author{
Valéria Cid Maia ${ }^{1,3}$ \& Marcelo da Costa Souza ${ }^{2}$ \\ ${ }^{1}$ Departamento de Entomologia, Museu Nacional, Quinta da Boa Vista, \\ São Cristóvão, CEP 20940-040, Rio de Janeiro, RJ, Brazil \\ ${ }^{2}$ Departamento de Botânica, Museu Nacional, Quinta da Boa Vista, \\ São Cristóvão, CEP 20940-40, Rio de Janeiro, RJ, Brazil \\ ${ }^{3}$ Corresponding author: Valéria Cid Maia, e-mail: maiavcid@acd.ufrj.br
}

MAIA, V.C. \& SOUZA, M.C. Insect galls of the xeric vegetation of Ilha do Cabo Frio (Arraial do Cabo, RJ, Brazil). Biota Neotrop. 13(3): http://www.biotaneotropica.org.br/v13n3/en/abstract?inventory+bn02213032013

\begin{abstract}
The xeric vegetation of Ilha do Cabo Frio (Arraial do Cabo, RJ) was investigated for insect galls, in May, June and August of 2012, totalizing 24 hours of field work. Forty-five morphotypes of insect galls were found on 29 species of host plant (25 genera and 18 families). Asteraceae and Myrtaceae were the most galled plant families. Leaves were the most galled plant organ, with ca. $62 \%$ of the total, followed by stems (ca. $22 \%$ ). Globoid and fusiform galls were the most frequent with about $29 \%$ and $24 \%$, respectively. The majority was glabrous (ca. 91\%), and one-chambered (ca. 84\%). Gallers of 30 morphotypes were determined. They belong to Diptera (Cecidomyiidae), and Lepidoptera. The former was the most frequent. Larvae of Muscomorpha (Diptera) (probably inquilines) were found in a single gall morphotype; parasitoids (Hymenoptera) in seven (ca. 15\% of the total), and predators (pseudoscorpion) in a single one. As there is no previous data of insect galls of Ilha do Cabo Frio, all records presented here are new. The geographic distribution of nine species of gall midges (Diptera, Cecidomyiidae) is widened. For the first time, eleven plant species are recorded as host of galling insects, and 24 gall morphotypes are described.
\end{abstract}

Keywords: galling insects, interaction insect-plant, host plants, island.

MAIA, V.C. \& SOUZA, M.C. Galhas de insetos da vegetação xérica da Ilha do Cabo Frio (Arraial do Cabo, RJ, Brasil). Biota Neotrop. 13(3): http://www.biotaneotropica.org.br/v13n3/pt/abstract?inventory+ bn02213032013

Resumo: A vegetação xérica da Ilha do Cabo Frio (Arraial do Cabo, RJ) foi investigada à procura de galhas de insetos em maio, junho e agosto de 2012, totalizando 24 horas de trabalho de campo. Quarenta e cinco morfotipos de galhas foram encontrados em 29 espécies de plantas hospedeiras (25 gêneros e 18 famílias). Asteraceae e Myrtaceae foram as famílias de planta com maior riqueza de galhas. Os órgãos vegetais mais galhados foram folha (cerca de $62 \%$ do total) e caule (cerca de 22\%). Galhas globóides e fusiformes foram mais frequentes com cerca de $29 \%$ e $24 \%$, respectivamente. A maioria era glabra (cerca de $91 \%$ ) e unilocular (cerca de $84 \%$ ). Os galhadores de 30 morfotipos foram determinados. Eles pertencem a Diptera (Cecidomyiidae) e Lepidoptera. Os primeiros foram mais frequentes. Larvas de Muscomorpha (Diptera) (provavelmente inquilinas) foram encontradas em um único morfotipo, parasitóides (Hymenoptera) em sete (cerca de 15\% do total) e predadores (pseudoscorpiões) em um. Como não havia informação prévia de galhas de insetos na Ilha do Cabo Frio, todos os registros apresentados aqui são novos. A distribuição geográfica de nove espécies de Cecidomyiidae (Diptera) é ampliada. Pela primeira vez, onze espécies de plantas são assinaladas como hospedeiras de galhas e 24 morfotipos de galhas são descritos. Palavras-chave: insetos galhadores, interação inseto-planta, plantas hospedeiras, ilha. 


\section{Introduction}

Galls are abnormal growth of plant tissues in response to a specific stimulus, usually produced by insects (Shorthouse \& Rohfritsch 1992). From an evolutionary point of view, galls can be seen as adaptations that allowed some insect taxa to feed on high quality tissues, and protect themselves from natural enemies and harsh abiotic factors (Price et al. 1986). The majority of galling insects are host-plant and plant-organ specific, and gall morphology is specific to each inducer (Floate et al. 1996).

The main objective of this paper is to elaborate an inventory of the gall insects of Ilha do Cabo Frio (Arraial do Cabo, RJ, Brazil). Ilha do Cabo Frio is localized in the municipality of Arraial do Cabo, Far East of the State of Rio de Janeiro. The island has an elongate shape and spreads over an area of ca. $6.5 \mathrm{~km}^{2}$. The highest altitude is $390 \mathrm{~m}$ above sea level. The climate is semi-arid, with average temperature varying from $20^{\circ}$ to $26^{\circ} \mathrm{C}$ and $800 \mathrm{~mm}$ of annual rain fall. It is part of the Cabo Frio Region, an important centre of plant diversity of the Neotropical region, due to the presence of several endemic taxa and unique plant communities, which are related to both a dry climate and a heterogeneous physical environment (Bohrer et al. 2009). Fabaceae, Myrtaceae, Euphorbiaceae, Nyctaginaceae, Sapotaceae, and Sapindaceae are the most common plant families in the Cabo Frio Region (Farág 1999, Rizzini 1987). The vegetation of the Ilha do Cabo Frio is classified as dry seasonal forest (Bohrer et al. 2009), and includes remnantes of the caatinga (Ururahy 1987). Nowadays, the island is under the Brazilian Navy control.

\section{Material and Methods}

Insect galls were collected in May, June and August, 2012 along the pathway to the new beacon $\left(23^{\circ} 00^{\prime} 11^{\prime \prime} \mathrm{S}\right.$ and $\left.42^{\circ} 00^{\prime} 21^{\prime \prime} \mathrm{WO}\right)$. The plants were investigated for insect galls during eight hours per month, totalizing 24 hours. All organs were examined, except for the subterraneous roots. Samples of host plants, preferably in the fertile state, were pressed in the field and later identified by MCS. The spelling of botanical species names and authorship were verified in http://www.theplantlist.org (accessed on 28 of November, 2012).

All gall morphotypes were photographed in field, using a digital photographic camera. Each morphotype was characterized based on shape (according to Isaias et al. 2013), color, presence or absence of trichomes, and number of internal chambers.

Immature insects were obtained by dissection of each kind of gall under a stereoscopic microscope. Pupal exuviae and adults were obtained by keeping samples of each gall morphotype individually in plastic pots layered at the bottom with damp cotton and covered by fine screening. All pots were checked daily for adults' emergence.

All insects were preserved in $70 \%$ alcohol. The gall midges (Diptera: Cecidomyiidae) were later mounted on slides for microscope, following the methodology of Gagné (1994). The genera were determined based on the keys of Gagné (1994), and species were identified based on gall shape, host plant and original descriptions. All material is deposited in the collection of Museu Nacional (MNNRJ)/ Universidade Federal do Rio de Janeiro.

\section{Results}

In Ilha do Cabo Frio (Arraial do Cabo, RJ), 45 morphotypes of insect galls were found on 29 species of host plant (25 genera and 18 families) (Table 1). Asteraceae was the most galled plant family, followed by Myrtaceae, with seven and six gall morphotypes, respectively. The medium number of galls per host species was 1.55.

Galls were recorded in leaves, stems, buds, tendrils and inflorescences. Leaves were the most galled plant organ, with ca. $62 \%$ of the total of the gall morphotypes, followed by stem (ca. $22 \%$ )
(Table 2). Otherwise, galls on fruits and aerial roots were not found. All morphotypes occurred in a single plant organ, excepting by the fusiform galls on Tournefortia membranacea DC. (Boraginaceae), which were induced on stems and leaf petioles.

The gall morphotypes were classified according to their shape in globoid (Figures 10, 14, 15, 18, 21, 25, 27, 31, 37, 39, 40, 43, and 45), fusiform (Figures 1, 2, 4, 6, 9, 16, 19, 22, 28, 41, 44), lenticular (Figures 3, 7, 8, 29, 34, 36), linear (Figures 5, 13), marginal roll (Figures 11, 17, 23, 32), ovoid (Figures 12, 26, 30), leaf roll (Figures 20, 24), cylindrical (Figure 33), deformed leaf (Figure 35), rosette (Figure 38), and clavate (Figure 42). The globoid and fusiform shapes were the most frequent ones, with about $28 \%$ and $24 \%$, respectively (Table 3 ). The majority was glabrous (41 morphotypes, ca. 91\%), being the remainders (4 morphotypes, ca. 09\%) hairy. About 84\% (38 morphotypes) were one-chambered, being 13\% (06 morphotypes) multi-chambered. A single morphotype was one or multi-chambered.

The gall inducing insects of 30 morphotypes was determined: nine were identified in species, four in genera, 10 in family, and seven in order. The gallers belong to two insect orders: Diptera (Cecidomyiidae), and Lepidoptera. The former was the most frequent galling taxa, being responsible for 23 morphotypes $(77 \%$ of the determined gallers) (Table 4), followed by Lepidoptera (ca. 23\%). The galler of fifteen morphotypes cannot be determined, because the gall samples were collected without dwellers or occupied by parasitoids and predators.

Larvae of Muscomorpha (Diptera) (probably inquilines) were found in a single gall morphotype on Selenicereus setaceus (Salm-Dyck) A. Berger ex Werderm. (Cactaceae), whose inducer is Neolasioptera cerei (Rübsaamen, 1905) (Diptera: Cecidomyiidae).

Parasitoids (Hymenoptera) were obtained from seven morphotypes (ca. 15\% of the total), and a predator (pseudoscorpion) from marginal leaf rolls of Handroanthus sp. (Bignoniaceae). This is the first record of pseudoscorpions in galls on Bignoniaceae.

As there is no previous data of insect galls of Ilha do Cabo Frio, all records presented here are new. The geographic distribution of nine species of gall midges (Diptera, Cecidomyiidae) is widened to the municipality of Arraial do Cabo (RJ): Lopesia similis Maia, 2004; Neolasioptera cerei (Rübsaamen, 1905); Clinodiplosis profusa Maia, 2001; Neolasioptera eugeniae Maia, 1993; Bruggmannia elongata Maia \& Couri, 1993; Pisphondylia brasiliensis Couri \& Maia, 1992; Proasphondylia guapirae Maia, 1993; Asphondylia communis Maia \& Couri, 1993 and Schismatodiplosis lantanae Rübsaamen, 1916.

Eleven plant species are recorded for the first time as host of galling insects: Baccharis bifrons Baker, Cyrtocymura scorpioides (Lam.) H.Rob. (Asteraceae), Tournefortia membranacea DC. (Boraginaceae), Capparis flexuosa (L.) L. (Capparaceae), Dalechampia micromeria Baill. (Euphorbiaceae), Senegalia polyphylla (DC.) Britton \& Rose (Fabaceae), Cissampelos pareira L. (Menispermaceae), Heteropterys chrysophylla (Lam.) DC. (Malpighiaceae), Eugenia candolleana DC., Eugenia punicifolia (Kunth) DC. (Myrtaceae), and Paullinia racemosa Wawra. Twenty-four gall morphotypes are described for the first time, the remainder was already known from other localities.

This is the second inventory of insect galls in Brazilian islands. The first one was developed in an protected area of Ilha Grande (Angra dos Reis, RJ), where 36 gall morphotypes were recorded on 22 plant species distributed in 16 families, being Myrtaceae and Asteraceae the most richest in number of gall morphotypes (Maia \& Oliveira, 2010).

The following description of the insect galls were presented here under host plant families, genera and species in alphabetical order. They included gall morphological characterization, associated fauna and previous records in coastal areas of the Southeastern region of Brazil. 
Table 1. Distribution of the number of insect gall morphotypes per host plant family, and species in Ilha do Cabo Frio (Arraial do Cabo, RJ, Brazil).

\begin{tabular}{|c|c|c|}
\hline Host Plant & & \\
\hline Family $(n=18)$ & Species $(n=29)$ & Number of insect gall morphotypes $(n=45)$ \\
\hline \multirow[t]{2}{*}{ Anacardiaceae } & Schinnus terebinthifolium Raddi & 01 \\
\hline & & Total: 01 \\
\hline \multirow[t]{5}{*}{ Asteraceae } & Baccharis bifrons Baker & 03 \\
\hline & Cyrtocymura scorpioides (Lam.) H.Rob. & 01 \\
\hline & Dasyphyllum sp. nov. & 02 \\
\hline & Trixis sp. & 01 \\
\hline & & Total: 07 \\
\hline \multirow[t]{3}{*}{ Bignoniaceae } & $\begin{array}{l}\text { Fridericia } \\
\text { conjugata (Vell.) L. G. Lohmann }\end{array}$ & 01 \\
\hline & Handroanthus sp. & 02 \\
\hline & & Total: 03 \\
\hline \multirow[t]{3}{*}{ Boraginaceae } & Cordia curassavica (Jacq.) Roem. \& Schult. & 02 \\
\hline & Tournefortia membranacea DC. & 03 \\
\hline & & Total: 05 \\
\hline \multirow[t]{2}{*}{ Burseraceae } & Protium heptaphylum (Aubl.) Marchand & 01 \\
\hline & & Total: 01 \\
\hline \multirow[t]{2}{*}{ Cactaceae } & Selenicereus setaceus (Salm-Dyck) A. Berger ex Werderm. & 01 \\
\hline & & Total: 01 \\
\hline \multirow[t]{2}{*}{ Capparaceae } & Capparis flexuosa (L.) L. & 01 \\
\hline & & Total: 01 \\
\hline \multirow[t]{2}{*}{ Erythroxylaceae } & Erythroxylum. ovalifolium Peyr. & 02 \\
\hline & & Total: 02 \\
\hline \multirow[t]{3}{*}{ Euphorbiaceae } & Croton compressus Lam. & 02 \\
\hline & Dalechampia micromeria Baill. & 01 \\
\hline & & Total: 03 \\
\hline \multirow[t]{3}{*}{ Fabaceae } & Copaifera cfr.sp. & 01 \\
\hline & Senegalia polyphylla (DC.) Britton \& Rose & 01 \\
\hline & & Total: 02 \\
\hline \multirow[t]{2}{*}{ Lamiaceae } & Not det. & 01 \\
\hline & & Total: 01 \\
\hline \multirow[t]{2}{*}{ Malpighiaceae } & Heteropterys chrysophylla (Lam.) DC. & 01 \\
\hline & & Total: 01 \\
\hline \multirow[t]{2}{*}{ Menispermaceae } & Cissampelos pareira $\mathrm{L}$. & 01 \\
\hline & & Total: 01 \\
\hline \multirow[t]{5}{*}{ Myrtaceae } & Eugenia candolleana DC. & 01 \\
\hline & Eugenia punicifolia (Kunth) DC. & 01 \\
\hline & Eugenia sulcata Spring ex Mart & 01 \\
\hline & Eugenia uniflora $\mathrm{L}$. & 03 \\
\hline & & Total: 06 \\
\hline \multirow[t]{3}{*}{ Nyctaginaceae } & Guapira opposita (Vell.) Reitz. & 04 \\
\hline & Guapira pernambucensis (Casar.) Lundell & 01 \\
\hline & & Total: 05 \\
\hline \multirow[t]{2}{*}{ Olacaceae } & Ximenia americana $\mathrm{L}$. & 01 \\
\hline & & Total: 01 \\
\hline \multirow[t]{2}{*}{ Sapindaceae } & Paullinia racemosa Wawra & 01 \\
\hline & & Total: 01 \\
\hline \multirow[t]{3}{*}{ Verbenaceae } & Lantana camara $\mathrm{L}$. & 02 \\
\hline & Lantana lilacina Desf. & 01 \\
\hline & & Total: 03 \\
\hline
\end{tabular}




\section{Anacardiaceae}

\section{Schinnus terebinthifolium Raddi}

Fusiform stem swelling, brown, glabrous, one-chambered (Figure 1). Galler: Lepidoptera. Galls collected in August. Previous records: Bertioga (SP), Grumari and Cabo Frio (Rio de Janeiro, RJ). Refs.: Maia et at. (2008), Oliveira \& Maia (2005), Maia (2013).

Table 2. Distribution of insect gall morphotypes per plant organ in Ilha do Cabo Frio (Arraial do Cabo, RJ, Brazil).

\begin{tabular}{cc}
\hline Galled plant organ & $\begin{array}{c}\text { Number of insect gall } \\
\text { morphotypes (n=45) }\end{array}$ \\
\hline Leaf & $28(62.2 \%)$ \\
Stem & $10(22.22 \%)$ \\
Bud & $05(11.11 \%)$ \\
Inflorescence & $01(2.22 \%)$ \\
Tendril & $01(2.22 \%)$ \\
Stem and petiole (leaf) & $01(2.22 \%)$ \\
\hline
\end{tabular}

\section{Asteraceae}

Baccharis bifrons Baker (new record of host plant)

Fusiform vein swelling, green or yellowish, glabrous, onechambered (Figure 2). Galler: Cecidomyiidae (Diptera) (1 larva de Cecidomyiidae on 23/V/2012). Galls collected in May. New gall morphotype.

Lenticular leaf gall, yellow, glabrous, one-chambered (Figure 3). Galler: not determined. Associated fauna: parasitoid (Hymenoptera: 1 adult on 25.VI.2012). Galls collected in May and June. New gall morphotype.

Fusiform stem swelling, green, glabrous, one-chambered (Figure 4). Galler: Lepidoptera. Galls collected in August. New gall morphotype.

Cyrtocymura scorpioides (Lam.) H.Rob. (new record of host plant)

Linear leaf gall, green, glabrous, one-chambered (Figure 5). Galler: Lepidoptera. Galls collected in May and June. New gall morphotype.

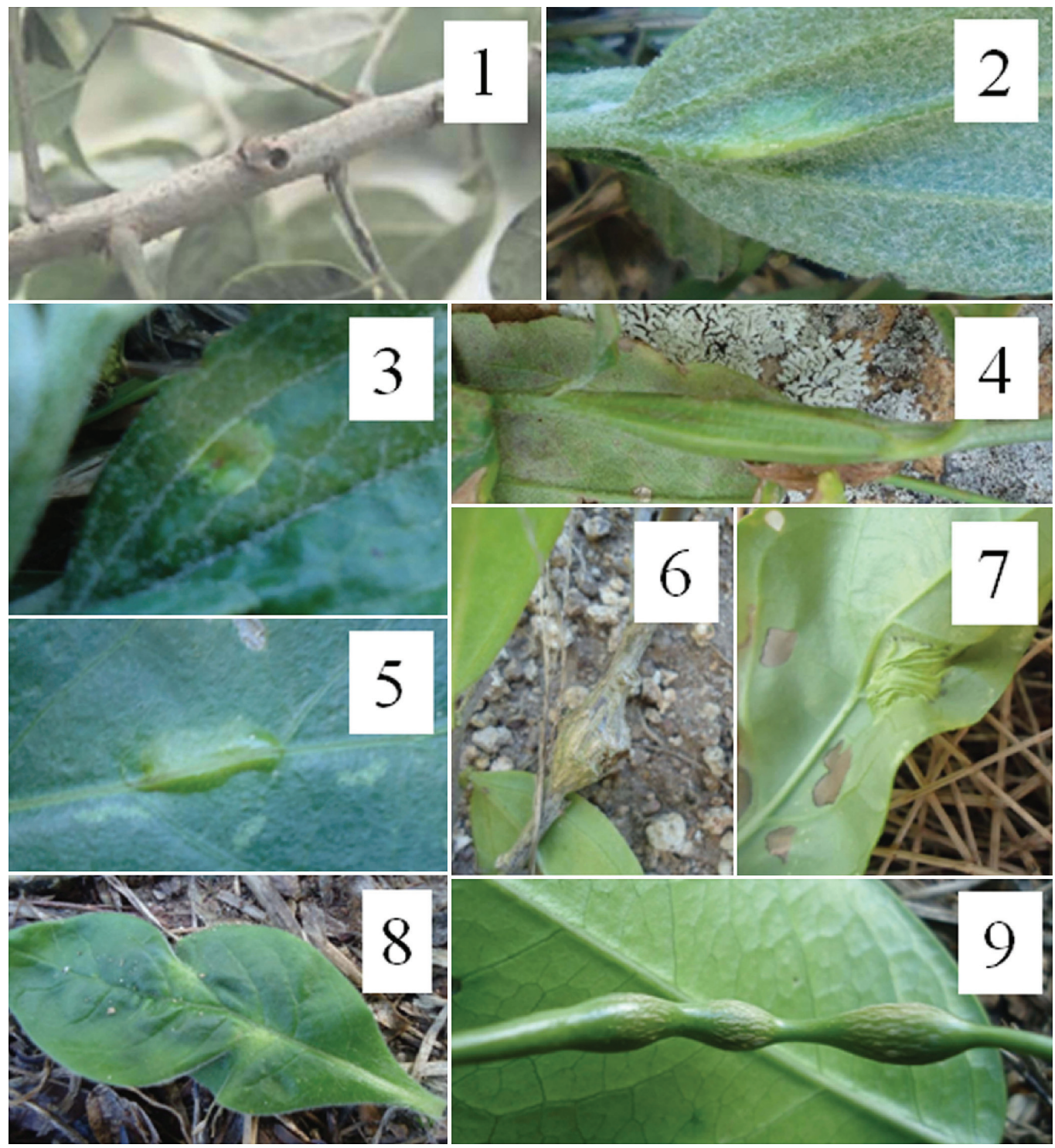

Figures 1-9. Insect galls of Ilha do Cabo Frio (Arraial do Cabo, RJ). 1) Stem gall on Schinnus terebinthifolium, 2) Vein gall on Baccharis bifrons, 3) Lenticular leaf gall on B. bifrons, 4) Stem swelling on B. bifrons, 5) Leaf gall on Cyrtocymura scorpioides, 6) Stem swelling on Dasyphyllum sp. nov., 7) Leaf gall on Dasyphyllum sp. nov., 8) Leaf gall on Trixis sp. nov., 9) Stem swelling on Fridericia conjugata. 


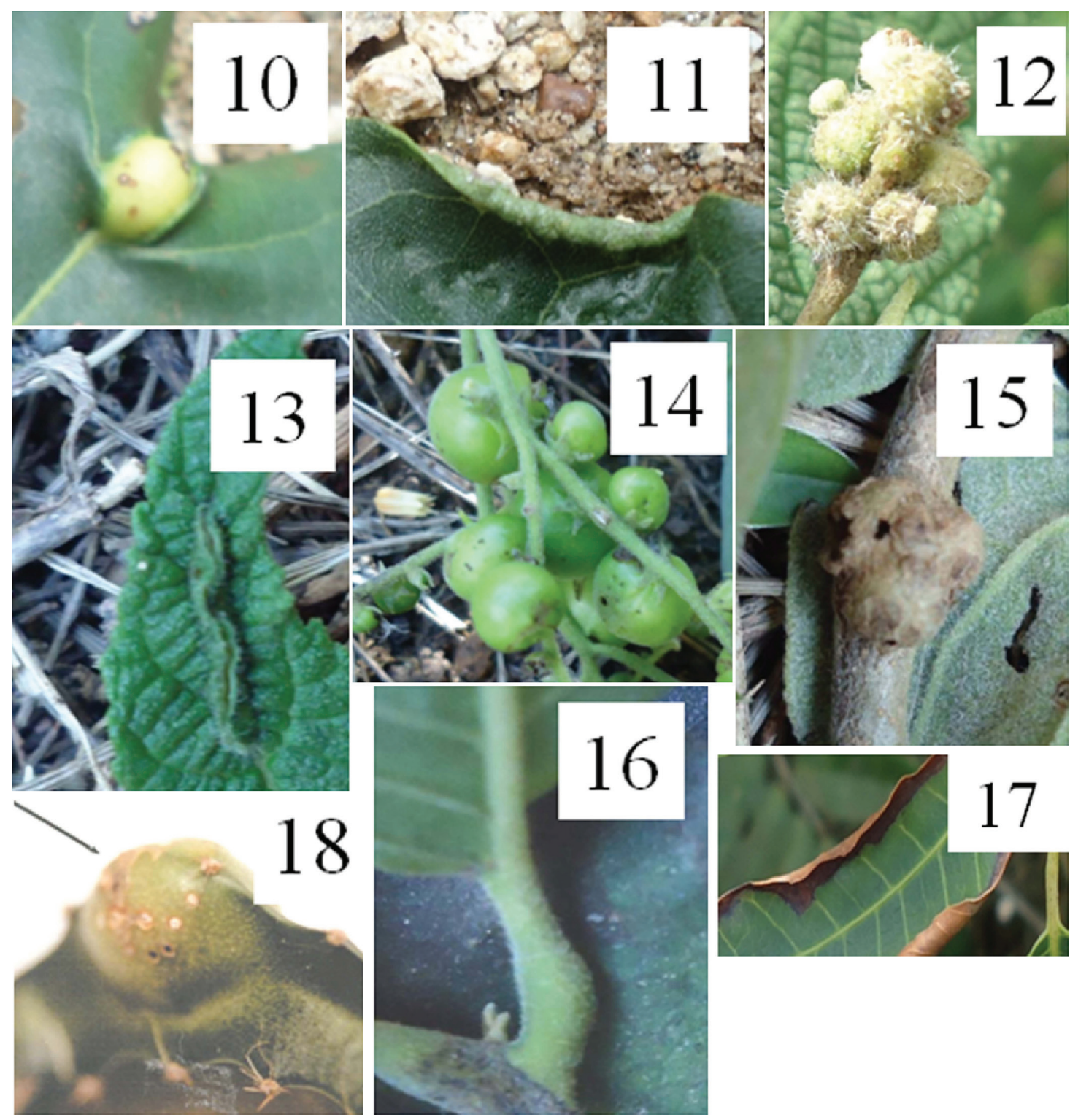

Figures 10-18. Insect galls of Ilha do Cabo Frio (Arraial do Cabo, RJ). 10) Globoid vein swelling on Handroanthus sp., 11) Marginal leaf roll on Handroanthus sp., 12) Inflorescence gall on Cordia curassavica, 13) Linear leaf gall on C. curassavica, 14) Bud gall on Tournefortia membranacea, 15) Stem swelling on T. membranacea, 16) Petiole swelling on T. membranacea., 17) Marginal leaf roll on Protium heptaphylum., 18) Stem swelling on Selenicereus setaceus.

Table 3. Distribution of insect gall morphotypes by shape in Ilha do Cabo Frio (Arraial do Cabo, RJ, Brazil).

\begin{tabular}{cc}
\hline Gall shape & $\begin{array}{c}\text { Number of insect gall } \\
\text { morphotypes (n=45) }\end{array}$ \\
\hline Globoid & $13(28.89 \%)$ \\
Fusiform & $11(24.44 \%)$ \\
Lenticular & $06(13.33 \%)$ \\
Linear & $02(4.44 \%)$ \\
Marginal roll & $04(8.89 \%)$ \\
Ovoid & $03(6.66 \%)$ \\
Leaf roll & $02(4.44 \%)$ \\
Cylindrical & $01(2.22 \%)$ \\
Rosette & $01(2.22 \%)$ \\
Clavate & $01(2.22 \%)$ \\
Deformed leaf & $01(2.22 \%)$ \\
\hline
\end{tabular}

Table 4. Distribution of insect gall morphotypes by galling insect order in Ilha do Cabo Frio (Arraial do Cabo, RJ, Brazil).

\begin{tabular}{cc}
\hline Galling insect order & $\begin{array}{c}\text { Number of gall morphotypes } \\
(\mathbf{n = 3 0 )}\end{array}$ \\
\hline Diptera (Cecidomyiidae) & $23(77 \%)$ \\
Lepidoptera & $07(23 \%)$ \\
\hline
\end{tabular}

Dasyphyllum sp. (new record of host plant)

Fusiform stem swelling, brown, glabrous, one-chambered (Figure 6). Galler: not determined. Galls collected on May and August. New gall morphotype.

Lenticular leaf gall, green, green, glabrous, rugose, onechambered (Figure 7). Galler: Lepidoptera (3 caterpillars on 27-28/ VIII/2012). Galls collected in August. New gall morphotype. 


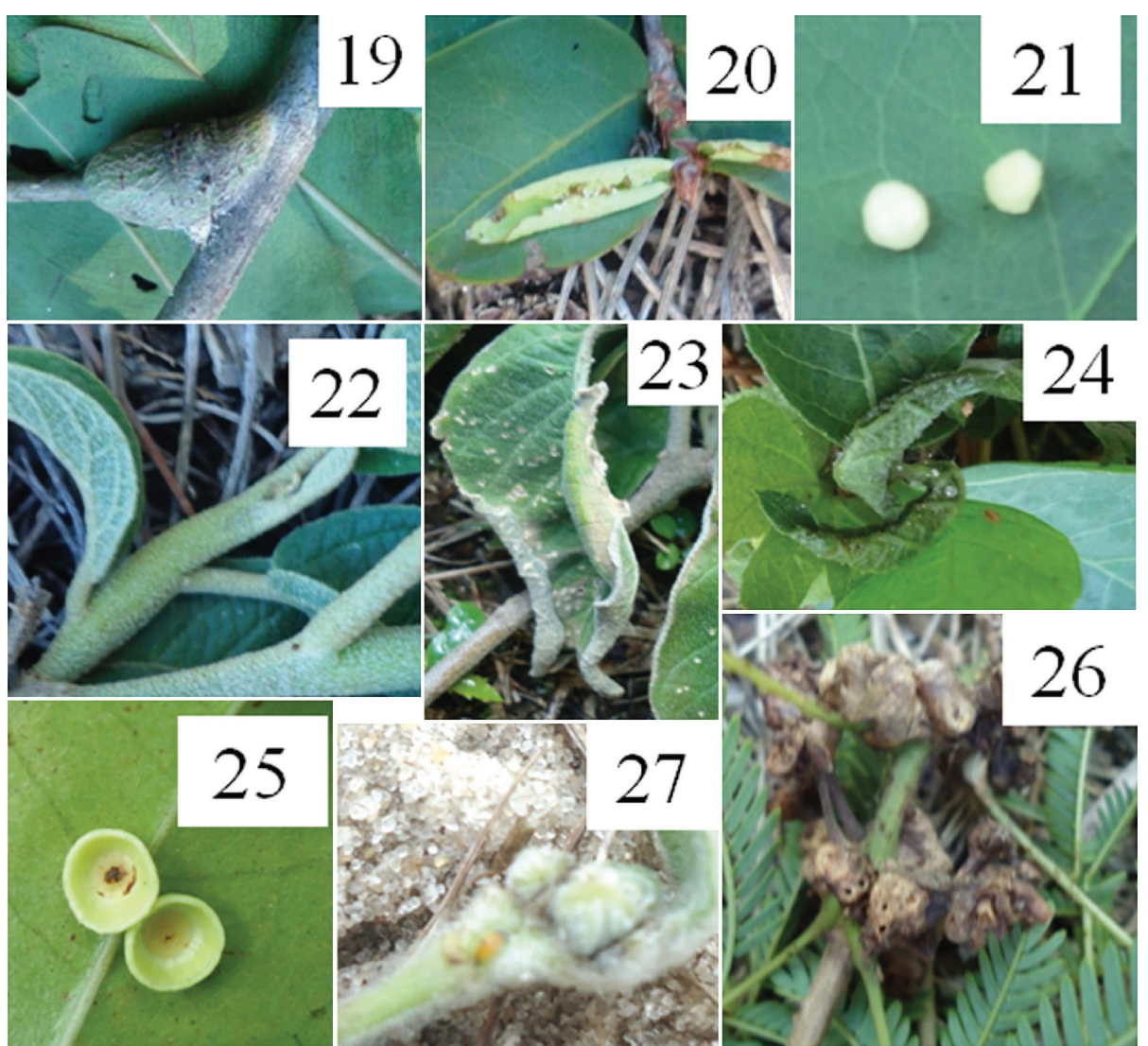

Figures 19-27. Insect galls of Ilha do Cabo Frio (Arraial do Cabo, RJ). 19) Stem swelling on Capparis flexuosa, 20) Young leaf roll on Erythroxylum ovalifolium, 21) Leaf gall on E. ovalifolium, 22) Petiole swelling on Croton compressus, 23) Marginal leaf roll on C. compressus, 24) Leaf roll on Dalechampia micromeria, 25) Leaf gall on Copaifera cfr. sp., 26) Bud gall on Senegalia polyphylla, 27) Bud gall on Lamiaceae (not determined).

Trixis sp. (new record of host plant)

Lenticular leaf gall, green, glabrous, one-chambered (Figure 8). Galler: not determined. Galls collected on June. New gall morphotype.

\section{Bignoniaceae}

\section{Fridericia conjugata (Vell.) L. G. Lohmann}

Fusiform tendril swelling, green, glabrous, one-chambered (Figure 9). Galler: Neolasioptera sp. (Diptera, Cecidomyiidae) (4 larvae on 26.VI.2012). Galls collected in June and August. Previous records: Maricá, Carapebus, Arraial do Cabo and Grumari (RJ) as Arrabidaea conjugata (Vell.) Mart. Refs.: Maia 2001, Monteiro et al. (1994), Oliveira \& Maia (2005).

\section{Handroanthus sp.}

Globoid leaf vein swelling, green or yellowish, glabrous, onechambered (Figure 10). Galler: Cecidomyiidae (Diptera). Associated fauna: parasitoids (2 larvae of Hymenoptera on 25.VI.2012). Galls collected in May, June and August.

Marginal leaf roll, green, glabrous, one-chambered (Figure 11). Galler: not determined. Associated fauna: predator (1 pseudoscorpion on 23/V/2012). Galls collected in May, June and August.

\section{Boraginaceae}

Cordia curassavica (Jacq.) Roem. \& Schult.

Inflorescence ovoid gall, green, hairy, one-chambered (Figure 12). Galler: Asphondylia sp. (Diptera, Cecidomyidae) (1 pupal exuvia on 22/V/2012). Galls collected in May, June and July. Previous records: Bertioga (SP), Maricá, Carapebus and Cabo Frio (RJ). Refs.: Maia et al. (2008), Maia (2001, 2013).

Linear leaf gall, green, glabrous, one-chambered (Figure 13). Galler: Not determined. Galls collected in June and July. New record of gall morphotype.

Tournefortia membranacea DC. (new record of host plant)

Globoid bud gall, green, glabrous, one-chambered (Figure 14). Galler: Cecidomyiidae (Diptera) (3 larvae on 22/V/2012, 1 larva and fragments of pupal exuviae on 27-28/VIII/2012). Associated fauna: inquiline (Lepidoptera: 1 caterpillar on 22/V/2012), and parasitoids (Hymenoptera: 5 pupae on 22.V.2012). Galls collected in May, June and August. New gall morphotype.

Globoid stem swelling, brown, glabrous, one or multi-chambered (Figure 15). Galler: Asphondylia sp. (Diptera, Cecidomyiidae) (1 pupa on 27-28/VIII/2012). Associated fauna: parasitoids (Hymenoptera: 3 pupae and 1 larva on 27-28/VIII/2012). Galls collected in June and August. New gall morphotype.

Fusiform stem and petiole swelling, green or brown, glabrous, one-chambered (Figure 16). Galler: not determined. Galls collected in June. New gall morphotype.

\section{Burseraceae}

Protium heptaphylum (Aubl.) Marchand

Marginal leaf roll, green, glabrous, one-chambered (Figure 17). Galler: Lopesia similis Maia, 2004 (Diptera, Cecidomyidae). Galls collected in May and June. Previous records: Carapebus (RJ). Ref.: Maia (2001). 


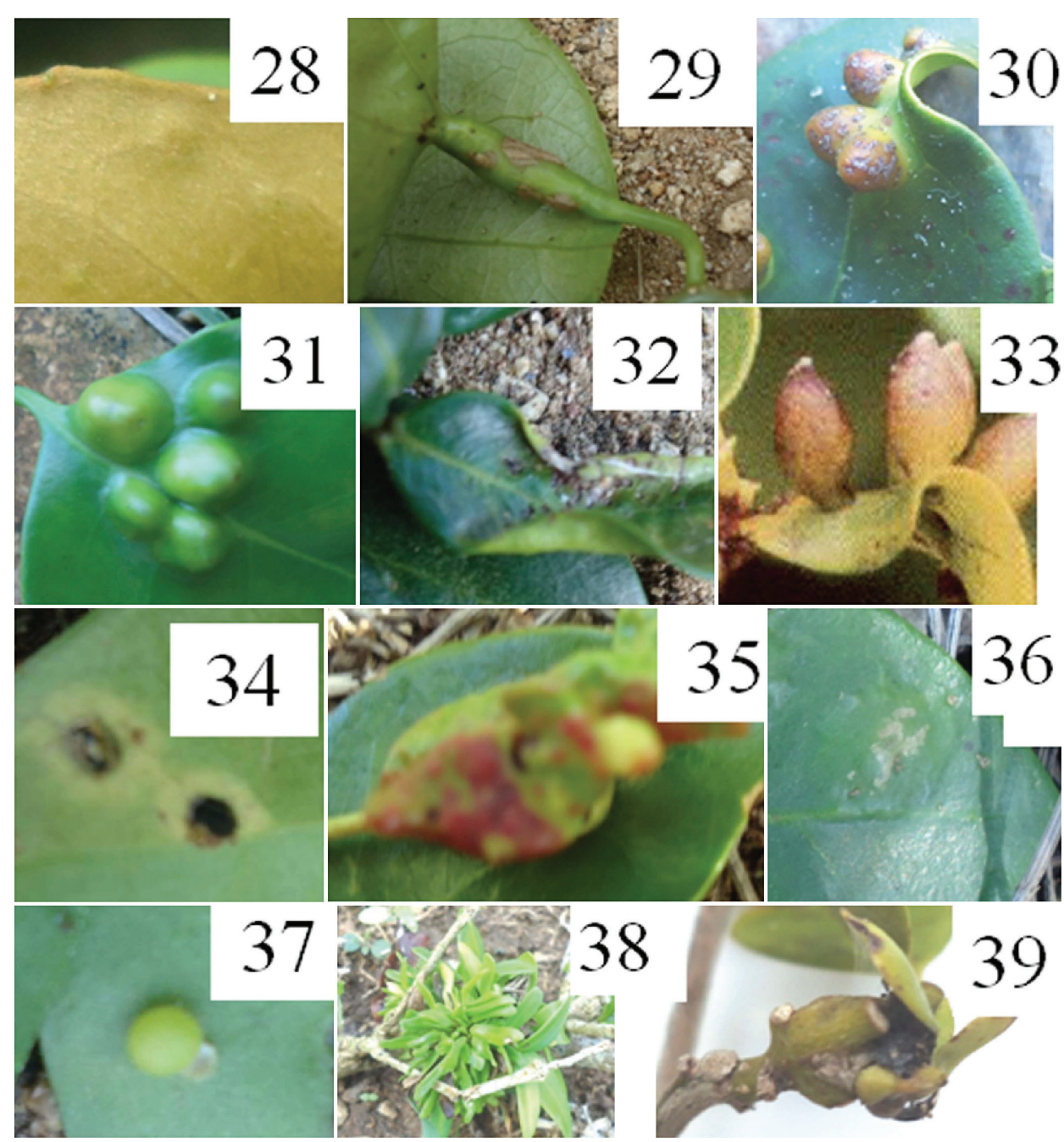

Figures 28-39. Insect galls of Ilha do Cabo Frio (Arraial do Cabo, RJ). 28) Leaf gall on Heteropterys chrysophylla, 29) Petiole swelling on Cissampelos pareira, 30) Leaf gall on Eugenia candolleana, 31) Leaf gall on Eugenia punicifolia, 32) Marginal leaf roll on Eugenia sulcata, 33) Cylindrical leaf gall on Eugenia uniflora, 34) Lenticular leaf gall on E. uniflora, 35) Deformed leaf on E. uniflora, 36) Lenticular leaf gall on Guapira opposita, 37) Globoid leaf gall on G. opposita, 38) Bud gall on G. opposita, 39) Stem swelling on G. opposita.

\section{Cactaceae}

Selenicereus setaceus (Salm-Dyck) A. Berger ex Werderm.

Globoid stem swelling, green glabrous, multi-chambered (Figure 18). Galler: Neolasioptera cerei (Rübsaamen, 1905) (Diptera: Cecidomyiidae). Associated fauna: 2 larvae of Muscomorpha on 23/V/2012. Galls collected in May and August. Previous records: Reserva Biológica Estadual da Praia do Sul (Ilha Grande, Angra dos Reis), and Maricá (RJ). Refs.: Maia \& Oliveira (2010), Maia (2001).

\section{Capparaceae}

Capparis flexuosa (L.) L. (new record of host plant)

Fusiform stem swelling, brown, glabrous, one-chambered (Figure 19). Galler: Lepidoptera (1 catterpilar on 25/VI/2012). Galls collected in June. New gall morphotype.

\section{Erythroxylaceae}

\section{Erythroxylum ovalifolium Peyr.}

Young leaf roll, green, glabrous, one-chambered (Figure 20). Galler: Clinodiplosis sp. (Diptera: Cecidomyiidae). Galls collected in May and June. Previous records: Reserva Biológica Estadual da
Praia do Sul (Ilha Grande, Angra dos Reis), Maricá and Carapebus (RJ). Refs.: Maia \& Oliveira (2010), Maia (2001).

Globoid leaf gall, green or yellowish, glabrous, one-chambered (Figure 21). Galler: Cecidomyiidae (Diptera). Associated fauna: fragments of Hymenoptera on 25/VI/2012. Galls collected in June and August. New record of gall morphotype.

\section{Euphorbiaceae}

Croton compressus Lam.

Fusiform petiole swelling, yellowish, glabrous, one-chambered (Figure 22). Galler: Lepidoptera (2 caterpillars on 23/V/2012). Galls collected on May, June and August. Previous record: Cabo Frio (RJ). Ref.: Maia 2013.

Marginal leaf roll, green, glabrous, one-chambered (Figure 23). Galler: not determined. Galls collected in June and August. New record of gall morphotype.

Dalechampia micromeria Baill. (new record of host plant)

Leaf roll, green, glabrous, one-chambered (Figure 24). Galler: not determined. Galls collected in June and August. New gall morphotype. 


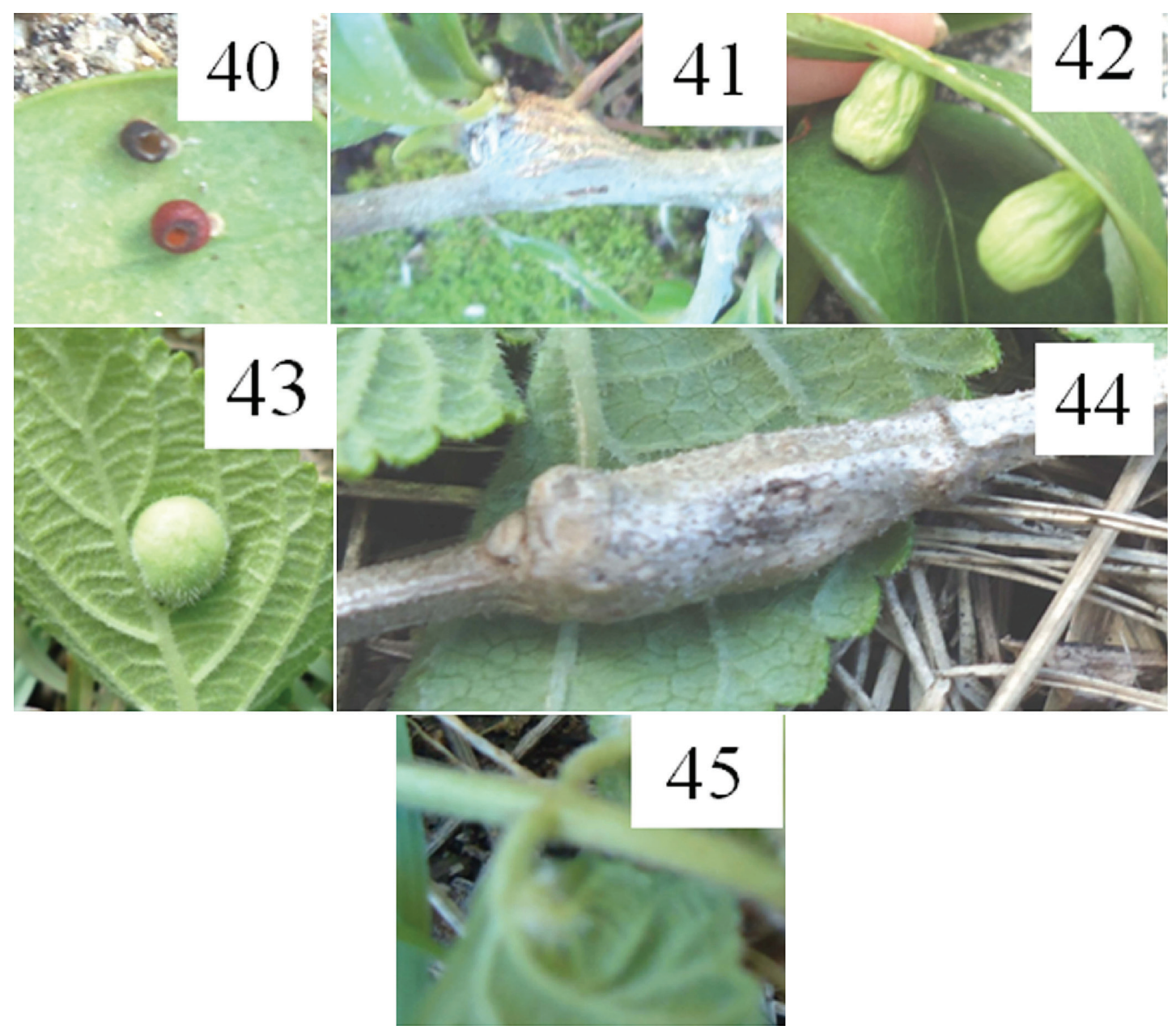

Figures 40-45. Insect galls of Ilha do Cabo Frio (Arraial do Cabo, RJ). 40) Leaf gall on Guapira pernambucensis, 41) Stem swelling on Ximenia americana, 42) Leaf gall on Paullinia racemosa, 43) Leaf gall on Lantana camara, 44) Stem swelling on L. camara, 45) Leaf gall on Lantana lilacina.

\section{Fabaceae}

\section{Copaifera sp.}

Globoid leaf gall, concave, yellow, glabrous, one-chambered (Figure 25). Galler: not determined. Galls collected in May.

Senegalia polyphylla (DC.) Britton \& Rose (new record of host plant)

Ovoid bud gall, green or brown, glabrous, multi-chambered (Figure 26). Galler: Cecidomyiidae (Diptera) (3 adults and 1 larva on 22/V/ 2012). Galls collected in May, June and August. New gall morphotype.

\section{Lamiaceae}

Not determined

Globoid apical bud gall, green, with white trichomes, onechambered (Figure 27) Galler: Cecidomyiidae (Diptera) (2 larvae on 25/VI/2012). Galls collected on May.

\section{Malpighiaceae}

Heteropterys chrysophylla (Lam.) DC. (new record of host plant)

Lenticular leaf gall (like a spot), brown, glabrous, one-chambered (Figure 28).Galler: not determined. Galls collected in May. New gall morphotype.

\section{Menispermaceae}

Cissampelos pareira L. (new record of host plant)

Fusiform petiole swelling, green, glabrous, multi-chambered (Figure 29). Galler: Cecidomyiidae (Diptera) (5 larvae on 27-28/ VIII/2012). Galls collected in August. New gall morphotype.

\section{Myrtaceae}

Eugenia candolleana DC. (new record of host plant)

Ovoid leaf gall, brown, glabrous, multi-chambered (Figure 30). Galler: not determined. Associated fauna: parasitoids (Hymenoptera, 02 larvae on 23/V/2012). Galls collected in May. New gall morphotype.

Eugenia punicifolia (Kunth) DC. (new record of host plant)

Globoid leaf gall, green, glabrous, one-chambered (Figure 31). Galler: not determined. Galls collected in June and August. New gall morphotype.

\section{Eugenia sulcata Spring ex Mart}

Marginal leaf roll, green, glabrous, one-chambered (Figure 32). Galler: not determined. Galls collected in June. New record of gall morphotype. Maia et al. 2008 recorded two bud galls on this plant species. 


\section{Eugenia uniflora L.}

Cylindrical leaf gall, green or red, glabrous, one-chambered (Figure 33). Galler: Clinodiplosis profusa Maia, 2001 (Diptera, Cecidomyidae). Galls collected in May, June and July. Previous records: Maricá and Grumari (RJ). Refs.: Maia (2001), Oliveira \& Maia (2005).

Lenticular leaf gall, yellowish peripherically and brown in the center, glabrous, one-chambered (Figure 34). Galler: Neolasioptera eugeniae Maia, 1993 (Diptera, Cecidomyidae). Galls collected in May, June and July. Previous records: Reserva Biológica Estadual da Praia do Sul (Ilha Grande, Angra dos Reis), Maricá and Grumari (RJ). Refs.: Maia \& Oliveira (2010), Maia (2001), Oliveira \& Maia (2005).

Deformed leaf, green or red, glabrous, one-chambered (Figure 35). Galler: not determined. Galls collected in June and July. New gall morphotype.

\section{Nyctaginaceae}

\section{Guapira opposita (Vell.) Reitz.}

Lenticular leaf gall, green, glabrous, one-chambered (Figure 36). Galler: Bruggmannia elongata Maia \& Couri, 1993 (Diptera, Cecidomyidae) (1 pupal exuviae on 22/V/2012). Galls collected in May, June and August. Previous records: Reserva Biológica Estadual da Praia do Sul (Ilha Grande, Angra dos Reis, (RJ), Maricá (RJ), Cabo Frio (RJ), Bertioga (SP). Refs.: Maia \& Oliveira (2010), Maia (2001, 2013), Maia et al. (2008).

Globoid leaf gall, green, yellow or red, glabrous, one-chambered (Figure 37). Galler: Cecidomyidae (Diptera ) (2 larvae and 1 pupal exuviae on 22/V/2012). Galls collected in May and June. Previous records: Bertioga (SP). Ref.: Maia et al. 2008.

Rosette bud gall, green, glabrous, multi-chambered (Figure 38). Galler: Pisphondylia brasiliensis Couri \& Maia, 1992 (Diptera, Cecidomyidae) (2 young larvae on 27-28/VIII/2012). Galls collected in May, June and August. Previous records: Bertioga (SP), and Maricá (RJ). Refs.: Maia et al. (2008), Maia (2001).

Globoid stem swelling, green, glabrous, one-chambered (Figure 39). Galler: Proasphondylia guapirae Maia, 1993 (Diptera, Cecidomyidae). Galls collected in August. Previous records: Reserva Biológica Estadual da Praia do Sul (Ilha Grande, Angra dos Reis), Bertioga (SP), Maricá (RJ). Refs.: Maia \& Oliveira (2010), Maia et al. (2008), Maia (2001).

\section{Guapira pernambucensis (Casar.) Lundell}

Globoid leaf gall, red, hairy, one-chambered (Figure 40). Galler: Cecidomyidae (Diptera) (1 pupal exuvia on 23/V/2012, and 2 adults on 26/VI/2012). Associated fauna: parasitoid (Hymenoptera: 1 adult on 26/VI/2012). Previous record: Parque Estadual Paulo César Vinha (Guarapari, ES). Ref.: Bregonci et al. (2010).

\section{Olacaceae}

\section{Ximenia americana $\mathrm{L}$.}

Fusiform stem gall, brown, glabrous, one-chambered (Figure 41). Galler: Asphondylia communis Maia \& Couri, 1993 (Diptera, Cecidomyiidae). Galls collected in June. Previous record: Maricá (RJ). Ref.: Maia (2001).

\section{Sapindaceae}

\section{Paullinia racemosa Wawra (new record of host plant)}

Clavate leaf gall, green, glabrous, one-chambered (Figure 42). Galler: Cecidomyiidae (Diptera). Galls collected in August.

\section{Verbenaceae}

\section{Lantana camara L.}

Globoid leaf gall, green, hairy, one-chambered (Figure 43). Galler: Schismatodiplosis lantanae Rübsaamen, 1916 (Diptera, Cecidomyidae) (3 third instar larvae in May, 2012 and 3 young larvae on 27-28/VIII/2012. Galls collected in May, June and August. Previous records: Maricá (RJ). Ref.: Maia (2001).

Fusiform stem swelling, brown, glabrous, multi-chambered (Figure 44). Galler: Lepidoptera. Galls collected in June and August. New gall morphotype.

\section{Lantana lilacina Desf.}

Globoid leaf vein swelling, green, hairy, one-chambered (Figure 45). Galler: Not determined. Associated fauna: parasitoid (Hymenoptera) (1 adult on 25.VI.2012). Galls collected in June. New gall morphotype.

\section{Discussion}

The vegetation of Ilha do Cabo Frio (Arraial do Cabo, RJ) presented 45 insect gall morphotypes. In other inventories in the Southeastern region of Brazil, the number of morphotypes varies a lot, being 132 the highest value and 36 the lowest (Table 5). As the frequency of the field works and the extension of the surveyed areas differed in these inventories, these values cannot be adequately compared. In spite of the methodological differences, the medium of gall morphotypes per host plant varied little (from 2.0 to 1.6). This result suggests that host plant species have a supporting capacity for galls (Table 5).

Asteraceae and Myrtaceae were pointed as the richest plant families in number of gall morphotypes. All other gall inventories in coastal areas of the Southern region of Brazil (Bertioga, Reserva Biológica Estadual da Praia do Sul in Ilha Grande, Grumari, Maricá, Carapebus, Arraial do Cabo, and Parque Estadual Paulo Cesar Vinha in Guarapari) indicated Myrtaceae as the most or one of the galled family, whereas Asteraceae were indicated only in two inventories (Bertioga and Reserva Biológica Estadual da Praia do Sul). The importance of Myrtaceae for restinga flora characterization is well known, being Eugenia the biggest genus in number of species (Rizzini 1979). Asteraceae are the one of the best represented plant family in Bertioga (Martins et al. 2008), differing from Reserva Biológica Estadual da Praia do Sul, where this family is represented by few species (Araújo \& Oliveira 1988). As the flora knowledge of the latter locality is still preliminary, Asteraceae can comprise more species than those already recorded. According to the plant diversity hypothesis (Fernandes 1992), the greatest richness of galls is showed by the most speciose plant families of each area. Our results corroborate this hypothesis.

Galls were found mainly in leaves. This is a world pattern pointed by Mani (1964) and corroborated in several galls inventories (Maia 2001, Maia \& Fernandes 2004, Maia et al. 2008, Maia 2011).

Fusiform and globoid galls were pointed as the most common in the present paper. The predominance of globoid galls has been also pointed in other inventories, as well as fusiform galls. Glabrous and one-chambered galls were also predominant (Maia 2001, 2011, Araújo et al. 2007, Bregonci et al. 2010, Carneiro et al. 2009), suggesting that this is the most common gall morphology in Brazilian biomes.

In spite of galling insects being found among six orders (Diptera, Lepidoptera, Coleoptera, Thysanoptera, Hemiptera and 
Insect galls of Ilha do Cabo Frio, RJ

Table 5. Number of insect gall morphotypes and medium of gall morphotypes per plant species in six localities of the Southeastern region of Brazil.

\begin{tabular}{lccc}
\hline \multicolumn{1}{c}{ Locality } & $\begin{array}{c}\text { Number of insect gall } \\
\text { morphotypes }\end{array}$ & $\begin{array}{c}\text { Medium of gall morphotypes } \\
\text { per plant species }\end{array}$ & Reference \\
\hline Bertioga (SP) & 132 & 1.9 & Maia et al. (2008) \\
Restinga de Maricá (RJ) & 75 & 1.9 & Maia (2001) \\
Parque Nacional de Jurubatiba (Carapebus, RJ) & 62 & 2.0 & Maia (2001) \\
Restinga do Grumari (Rio de Janeiro, RJ) & 43 & 1.7 & Oliveira \& Maia (2005) \\
Reserva Biológica Estadual da Praia do Sul & 36 & 1.6 & Maia \& Oliveira (2010) \\
(Illha Grande, Angra dos Reis, RJ) & & 1.8 & Bregonci et al. (2010) \\
Parque Estadual Paulo César Vinha (Guarapari, ES) & 38 & &
\end{tabular}

Table 6. Frequency of parasitoids associated with insect galls in two coastal areas of the Southeastern region of Brazil.

\begin{tabular}{lcc}
\hline \multicolumn{1}{c}{ Locality } & Frequency of parasitoids (\%) & Reference \\
\hline Bertioga (SP) & 48.0 & Maia et al. (2008) \\
Parque Estadual Paulo César Vinha (Guarapari, ES) & 31.5 & Bregonci et al. (2010) \\
Ilha do Cabo Frio (RJ) & 15.0 & Present study \\
\hline
\end{tabular}

Hymenoptera), only dipterous, and lepidopterous galls were recorded in Ilha do Cabo Frio. Diptera are the most frequent galling order in all zoogeographic regions (Felt 1940), Lepidoptera are the second in the Neotropical region (Maia 2006).

The occurrence of parasitoids, predators, inquilines and successors in insect galls is very common (Maia 2001, Maia \& Fernandes 2004). In Ilha do Cabo Frio, parasitoids were less frequent than in other surveyed areas of the State of Rio de Janeiro (Table 6). Otherwise, the presence of pseudoscorpions is very rare. Only two previous records are known in Brazil, one in bud galls induced by Stephomyia rotundifolium Maia, 1993 (Diptera, Cecidomyiidae) on Eugenia adstringens Cambess. (= Eugenia rotundifolia Casar) (Myrtaceae) and the other in stem galls induced by Houardodiplosis rochae Tavares, 1925 (Diptera, Cecidomyiidae) on Combretum leprosum Mart \& Eicher (Combretaceae). Besides, two records are known from Africa, both in stem galls of Poaceae (Judson \& Heurtault 1996).

Little is known about insect galls in Brazilian islands, as only two areas were investigated: Reserva Biológica Estadual da Praia do Sul, in Ilha Grande (Angra dos Reis, RJ), and Ilha do Cabo Frio (Arraial do Cabo, RJ). In both surveys, Asteraceae and Myrtaceae were the richest plant families in gall diversity. These families are well represented in these islands, suggesting that the plant richness is associated with gall diversity, as proposed by Southwood $(1960,1961)$.

\section{Conclusion}

The insect galls inventory of Ilha do Cabo Frio (Arraial do Cabo, RJ) corroborates the patterns already known in relation to galled plant organs, gall shape, galling taxa and host plant families. The geographic distribution of nine species of gall midges (Diptera, Cecidomyiidae) is widened. For the first time, eleven plant species are recorded as host of galling insects, pseudoscorpions are recorded in galls on Bignoniaceae, and 24 gall morphotypes are described.

\section{Acnowledgements}

We are grateful to the Brazilian Navy for infrastructure, Conselho Nacional de Desenvolvimento Científico e Tecnológico (CNPq) for financial support (Proc. 300237/2010-3) and Dr. Debora Medeiros (Museu Nacional) for Euphorbiaceae identification.

\section{References}

ARAÚJO, D. S. D. \& OLIVEIRA, R. R. 1988. Reserva Biológica Estadual da Praia do Sul (Ilha Grande, Estado do Rio de Janeiro): lista preliminar da flora. Acta Bot. Bras. 1(2):83-94. Suplemento.

ARAÚJO, W.S., GOMES-KLEIN, V.L. \& SANTOS, B.B. 2007. Galhas Entomógenas Associadas à Vegetação do Parque Estadual da Serra dos Pireneus, Pirenópolis, Goiás, Brasil. R. Bras. Bioci. 5(supl. 1):45-47.

BOHRER, C.B.A., DANTAS, H.G.R., CRONEMBERGER, F.M., VICENS, R.S. \& ANDRADE, S.F. 2009. Mapeamento da vegetação e do uso do solo no centro de diversidade vegetal de Cabo Frio, Rio de Janeiro, Brasil. Rodriguésia 60(1 01-023.

BREGONCI, J.M., POLYCARPO, P.V. \& MAIA, V.C. 2010. Insect galls of the Parque Estadual Paulo César Vinha (Guarapari, ES, Brazil). Biota Neotrop. 10(1): http://www.biotaneotropica.org.br/v10n1/en/abstract?in ventory+bn01410012010.

CARNEIRO, M.A.A., BORGES, R.A.X., ARAÚJO, A.P.A. \& FERNANDES, G.W. 2009. Insetos indutores de galhas da porção sul da Cadeia do Espinhaço, Minas Gerais, Brasil. Rev. Bras. Entomol. 53(4):570-592. http://dx.doi.org/10.1590/S0085-56262009000400007

FARÁG, P.R.C. 1999. Estrutura dos estratos arbóreo de mata litorânea semicaducifólia sobre solo arenoso no município de Búzios-RJ. Dissertação de Mestrado em Botânica, Universidade Federal do Rio de Janeiro, 87p.

FELT, E.P. 1940. Plant galls and gall makers. Ithaca, 364p.

FERNANDES, G.W. 1992. Plant family size and age effects on insular gallforming species richness. Glob. Ecol. Biogeogr. Let. 2:71-74. http:// dx.doi.org/10.2307/2997508

FLOATE, K.D., FERNANDES, G.W. \& NILSSON, J.A. 1996. Distinguishing intrapopulacional categories of plants by their insect faunas: galls on rabbitbrush. Oecologia 105:221-229.

GAGNÉ, R.J. 1994. The gall midges of the neotropical region. Cornell University Press, Ithaca, New York, 352p.

ISAIAS, R.M.S., CARNEIRO, R.G.S., OLIVEIRA, D.C. \& SANTOS, J.C. 2013. Illustrated and Annotated Checklist of Brazilian Gall Morphotypes. Neotrop Entomol. 42:230-239. PMid:23949804. http:// dx.doi.org/10.1007/s13744-013-0115-7

JUDSON, M. L. I. \& J. HEURTAULT. 1996. Nanolpium species (Garypoidea, Olpiidae) on grasses in southern Africa - a new niche for pseudoscorpions. Rev. Suisse Zool., ser. 321-326.

MAIA, V.C. 2001. The gall midges (Diptera, Cecidomyiidae) from three restingas of Rio de Janeiro State, Brazil. Rev. Bras. Zool. 18(2):583-629. http://dx.doi.org/10.1590/S0101-81752001000200028 
MAIA, V.C. 2006. Galls of Hemiptera, Lepidoptera and Thysanoptera from Central and South America. Pub. Avul. Mus. Nac. 110:01-24.

MAIA, V.C. 2011. Characterization of insect galls, gall makers, and associated fauna of Platô Bacaba (Porto de Trombetas, Pará, Brazil). Biota Neotrop. 11(4): http://www.biotaneotropica.org.br/v11n4/en/ abstract?article+bn00511042011.

MAIA, V.C. 2013. Galhas de insetos em restingas da região sudeste do Brasil com novos registros. Biota Neotrop. 13(1):183-209. http://dx.doi. org/10.1590/S1676-06032013000100021

MAIA, V.C. \& FERNANDES, G.W. 2004. Insect galls from Serra de São José (Tiradentes, MG, Brazil). Braz. J. Biol. 64(3):1-22.

MAIA, V.C. \& OLIVEIRA, J.C. 2010. Insect galls of the Reserva Biológica Estadual da Praia do Sul (Ilha Grande, Angra dos Reis, RJ). Biota Neotrop. 10(4): http://www.biotaneotropica.org.br/v10n4/en/abstract?inventory+ bn04110042010.

MAIA, V.C., MAGENTA, M.A.G. \& MARTINS, S.E. 2008. Ocorrência e caracterização de galhas de insetos em áreas de restinga de Bertioga (São Paulo, Brasil). Biota Neotrop. 8(1): http:/www.biotaneotropica. org.br/v8n1/PT

MANI, M.S. 1964. Ecology of plant galls. W. Junk, The Hague, 434p.

MARTINS, S.E., ROSS, L., SAMPAIO, P.S.P. \& MAGENTA, M.A.G. 2008. Caracterização florística de comunidades vegetais de restinga em Bertioga, SP, Brasil. Acta Bot. Bras. 22(1):249-274. http://dx.doi. org/10.1590/S0102-33062008000100024
MONTEIRO, R.F., FERRAZ, F.F.F., MAIA, V.C. \& AZEVEDO, M.A.P. 1994. Galhas entomógenas em restingas: uma abordagem preliminar. In Anais do III Simpósio de Ecossistemas da Costa Brasileira: subsídios a um gerenciamento ambiental (S. Watanabe, coord.). ACIESP, São Paulo, v.3, p.210-220.

OLIVEIRA, J.C. \& MAIA, V.C. 2005. Ocorrência e caracterização de galhas de insetos na restinga de Grumari (Rio de Janeiro, RJ, Brasil). Arq. Mus. Nac.1 63(4):669-675.

PRICE, P.W., WARING, G.L. \& FERNANDES, G.W. 1986. Hypotheses on the adaptive nature of galls. P. Entomol. Soc. Wash 88:361-363.

RIZZINI, C.T. 1997.Tratado de Fitogeografia do Brasil. 2. ed. São Paulo: Âmbito Cultural, 747p.

RIZZINI, C.T. 1979. Tratado de Fitogeografia do Brasil. São Paulo, Hucite, v.2, $54 \mathrm{p}$

SHORTHOUSE, J.D. \& ROHFRITSCH, O. 1992. Biology of insect induced galls. Oxford University Press, New York. PMCid:PMC1977397.

SOUTHWOOD, T.R.E. 1960. The abundance of the Hawaiian trees and the number of their associated insect species. Proc. Hawaii. Entomol. Soc. 17:299-303

SOUTHWOOD, T.R.E. 1961. The number of insect associated with various trees. J. Anim. Ecol. 30:1-8. http://dx.doi.org/10.2307/2109

URURAHY, J.C.C. 1987. Nota sobre uma formação fisionômica-ecológica disjunta da estepe nordestina na área do Pontal de Cabo Frio. Rev. Bras. Geogr. 49(4):25-29. http://dx.doi.org/10.2307/2109 